\title{
EDITORIAL
}

\section{Neurosurgery in the storm of COVID-19: suggestions from the Lombardy region, Italy (ex malo bonum)}

\author{
Marco Cenzato, MD, ${ }^{1}$ Francesco DiMeco, MD, ${ }^{2-4}$ Marco Fontanella, MD, ${ }^{5}$ Davide Locatelli, MD, ${ }^{6}$ and \\ Franco Servadei, MD $^{7}$
}

\begin{abstract}
1Department of Neurosurgery, Grande Ospedale Metropolitano Niguarda, Milan; ${ }^{2}$ Department of Pathophysiology and Transplantation University of Milan; ${ }^{3}$ Department of Neurosurgery, Fondazione IRCCS Istituto Neurologico Besta, Milan, Italy; ${ }^{4}$ Department of Neurological Surgery, Johns Hopkins Medical School, Baltimore, Maryland; ${ }^{5}$ Department of Neurosurgery, Spedali Civili and University of Brescia; ${ }^{6}$ Department of Neurosurgery, Ospedale di Circolo ASST Sette Laghi, University of Insubria, Varese; and 'Department of Neurosurgery, Humanitas Research Hospital and University, Milan, Italy
\end{abstract}

$\mathrm{O}$ N February 21, 2020, Italy's first patient infected by coronavirus was reported in Codogno (Lombardy). Standpoints among experts were then initially discordant, spanning from those who claimed that the illness was little more than the flu to those who advised it was a very dangerous disease. However, after 3 days, the number of cases increased to 221 and then up to 1337 on the 1st of March. A few weeks later, on March 21 there were 53,578 cases with 4825 deaths. From the very beginning, Lombardy has been the most affected region in Italy. As a matter of fact, in March 2020, the region of Lombardy (Italy's most populated region, accounting for 10,060,574 inhabitants) has found itself overwhelmed by the epidemic, initially quite unaware of the dramatic extent of this outbreak.

\section{The Hub-and-Spoke System}

Since about $10 \%$ of COVID-19 patients require admission to the ICU due to serious respiratory insufficiency, it soon became evident before the pandemic that the number of available ICU beds within the region (a total of 720) was by far not enough. Therefore, by applying various strategies, ${ }^{1}$ a tremendous effort has been put in place in order to increase the critical care capacity; as of this writing, there are about 1200 COVID-19 patients admitted to ICUs in our region.

These strategies to increase the regional ICU allowance included the reduction of all surgical activities starting with elective to ultimately also include emergency cases. The aim was to shift subspecialty ICU facilities and anesthesiologists to the care of COVID-19 patients. To this end, the regional government asked us to estimate the number of true neurosurgical emergency procedures and of nondeferrable elective ones for a foreseeable period of 2 months. From a quick survey of historical data from all neurosurgery departments in Lombardy, the estimated number of surgical emergencies was ranging between 80 and 100 per week for the entire region, 40 to 50 of whom were concentrated within the Milan metropolitan area. Approximately the same number of procedures was estimated for nondeferrable elective cases, mostly tumors.

According to such estimated caseloads, a plan of action was settled on clustering all urgent neurosurgical activities (mainly trauma and vascular) among only 3 centers (Niguarda, Brescia, and Varese), which were chosen based on geographical criteria as well as their receptive capacity, creating a hub-and-spoke scheme. ${ }^{2}$ Only one other hospital for the entire region (Fondazione IRCCS Istituto Neurologico Besta) was identified to deal with nondeferrable tumor cases.

\section{The Emergency Hubs}

All hub hospitals for emergencies have neurosurgical, trauma, stroke, and endovascular 24/7 coverage allowing them to handle all urgent cases. Neurosurgeons agreed to move from the spoke hospitals to the hub hospitals, which represents an innovative element compared with the usual competitive setting. Thanks to the cooperation and collaboration of more than 20 neurosurgeons from spoke hospitals and also some spine orthopedic surgeons, the number of on-duty neurosurgeons at hub hospitals was doubled. In addition, at each hub hospital, one on-call service of complex spinal surgery and one of vascular surgery are set up.

From the hub-and-spoke system establishment, all neurosurgical units in spoke hospitals gradually became inactive within a week. Thus, the number of neurosurgical departments managing emergencies was reduced in a very short period of time, from 15 down to 3 . Some minimal activity was occasionally still performed at spoke centers only for very critical cases. 
During the first 2 weeks of activity of the hub-andspoke system, the Niguarda Hospital admitted 95 emergency cases and operated on 47 patients, Brescia admitted 88 cases and operated on 23 patients, and Varese admitted 34 cases and operated on 13 patients, for a total of 83 surgical cases, a number lower than expected. This was mainly related to people being confined to their homes and a decrease in activities, leading to a significant reduction in trauma. Intriguingly, we also observed a reduction in subarachnoid hemorrhages (despite the change of season) and strokes, which hardly finds a straight explanation. So far, 15 COVID-19-positive patients have undergone surgery. Of these patients, 9 had a regular course, 5 patients died, and 1 patient is in critical condition, all because of pulmonary complications. When performing surgery on COVID-19 patients, all operators are required to protect themselves with double gloves, double surgical gowns, surgical N95 respirator masks, and disposable face shields. All surgeries on COVID-19 patients are conducted inside specifically dedicated operating rooms, and the patients are admitted to dedicated ICUs or dedicated wards depending on their status.

\section{The Neurooncological Hub}

In order to create the neurooncological hub, an action plan was generated and shared among hubs and spokes along with the creation of a web-based platform for conference calls and sharing radiological images. A patient waitlist was created that included exclusively nondeferrable tumor cases. Patients were categorized according to the severity of their illness into 3 classes: $\mathrm{A}++, \mathrm{A}+$, and A. "A++" includes patients requiring immediate surgical treatment as in the case of rapidly increasing intracranial pressure, impaired consciousness, and symptomatic medullary compression. "A+" refers to patients who require surgery within 7 to 10 days as for intracranial tumors with relevant mass effect and/or progressive neurological deficits. "A" encompasses patients with tumors causing neurological deficits and/or suspicion of malignant nature. This class owns the lower level of urgency requiring treatment within 1 month. A++ cases are performed at one of the 3 hubs with an emergency department or, in selected cases, at the spoke's site whenever possible. In contrast, A+ and A patients are referred to the neurooncological hub through an inter-institutional board reviewing process. Eligible patients are then scheduled at the neurooncological hub based on the urgency and availability of both the operating room slots and the referring surgical team. Due to the necessity to keep the neurooncological hub virus free, each patient undergoes additional screenings, namely, measurements of body temperature, $\mathrm{O}_{2}$ saturation, complete blood cell count, C-reactive protein, and transaminases; chest radiography; pharyngeal COVID swab; and a comprehensive interview focusing on COVID-19. In our experience, the presented scheme has proven highly effective. Tellingly, at the end of the 2nd operational week, 65 patients affected by highly complex, nondeferrable tumors were successfully operated on by both institutional and noninstitutional surgical teams at the Fondazione IRCCS Istituto Neurologico.

\section{Lessons Learned: Ex Malo Bonum}

In our albeit preliminary experience, opening the hospital doors to neurosurgeons coming from other institutions has offered an unprecedented opportunity of collaboration and integration of teams, which will ultimately serve as a model not only to cope with catastrophic events but also to establish a basis for a higher level of rationalization of neurosurgical patient care. Even though originating from ominous circumstances, it will definitely remain a learning experience for a better future: ex malo bonum!

https://thejns.org/doi/abs/10.3171/2020.3.JNS20960

\section{Acknowledgments}

We sincerely appreciate the collaboration of the following colleagues from spoke centers: Marco Locatelli, Lorenzo Bello, Marco Benazzo, Giannantonio Spena, Claudio Bernucci, Erik Sganzerla, Marcello Egidi, Diego Spagnoli, Carlo Giussani, Michele Incerti, Giorgio Lorusso, Roberto Stefini, Roberto Assietti, Silvio Bellocchi, Maurizio Fornari, Miran Skrap, and Fulvio Tartara.

\section{References}

1. Grasselli G, Pesenti A, Cecconi M. Critical care utilization for the COVID-19 outbreak in Lombardy, Italy: early experience and forecast during an emergency response [published online March 13, 2020]. JAMA. doi:10.1001/jama.2020.4031

2. Chieregato A, Volpi A, Gordini G, et al. How health service delivery guides the allocation of major trauma patients in the intensive care units of the inclusive (hub and spoke) trauma system of the Emilia Romagna Region (Italy). A cross-sectional study. BMJ Open. 2017;7(9):e016415.

\section{Disclosures}

The authors report no conflict of interest.

\section{Correspondence}

Francesco DiMeco: francesco.dimeco@istituto-besta.it.

\section{INCLUDE WHEN CITING}

Published online April 10, 2020; DOI: 10.3171/2020.3.JNS20960. 